\title{
Methylation of OPRL1 mediates the effect of psychosocial stress on binge drinking in adolescents
}

\begin{abstract}
Barbara Ruggeri, ${ }^{1,2}$ Christine Macare, ${ }^{1,2}$ Serena Stopponi, ${ }^{3}$ Tianye Jia, ${ }^{1,2}$ Fabiana M. Carvalho, ${ }^{1,2}$ Gabriel Robert, ${ }^{1,2}$ Tobias Banaschewski, ${ }^{4}$ Arun L.W. Bokde, ${ }^{5}$ Uli Bromberg, ${ }^{6}$ Christian Büchel, ${ }^{6}$ Anna Cattrell, ${ }^{1,2}$ Patricia J. Conrod, ${ }^{1,7}$ Sylvane Desrivières, ${ }^{1,2}$ Herta Flor, ${ }^{8}$ Vincent Frouin, ${ }^{9}$ Jürgen Gallinat, ${ }^{10}$ Hugh Garavan, ${ }^{5,11}$ Penny Gowland, ${ }^{12}$ Andreas Heinz, ${ }^{10}$ Bernd Ittermann, ${ }^{13}$ Jean Luc Martinot, ${ }^{14}$ Marie-Laure Paillère Martinot ${ }^{14}$ Frauke Nees, ${ }^{8}$ Dimitri Papadopoulos-Orfanos, ${ }^{9}$ Tomás Paus, ${ }^{12,15,16}$ Luise Poustka, ${ }^{4}$ Michael N. Smolka, ${ }^{17}$ Nora C. Vetter, ${ }^{17}$ Henrik Walter, ${ }^{10}$ Robert Whelan, ${ }^{18}$ Wolfgang H. Sommer, ${ }^{4}$ Georgy Bakalkin, ${ }^{19}$ Roberto Ciccocioppo, ${ }^{3}$ Gunter Schumann, ${ }^{1,2}$ and the IMAGEN consortium ${ }^{\dagger}$

${ }^{1}$ Institute of Psychiatry, Psychology and Neuroscience, King's College London, London, UK; ${ }^{2} \mathrm{MRC}$ Social, Genetic and Developmental Psychiatry (SGDP) Centre, London, UK; ${ }^{3}$ School of Pharmacy, University of Camerino, Camerino, Italy; ${ }^{4}$ Central Institute of Mental Health, Medical Faculty Mannheim, Heidelberg University, Mannheim, Germany; ${ }^{5}$ Institute of Neuroscience, Trinity College Dublin, Dublin, Ireland; ${ }^{6}$ Universitaetsklinikum Hamburg Eppendorf, Hamburg, Germany; ${ }^{7}$ Department of Psychiatry, Université de Montreal, Montreal, QC, Canada; ${ }^{8}$ Department of Cognitive and Clinical Neuroscience, Central Institute of Mental Health, Heidelberg University, Mannheim, Germany; ${ }^{9}$ Neurospin, Commissariat à l'Energie Atomique et aux Energies Alternatives, Paris, France; ${ }^{10}$ Department of Psychiatry and Psychotherapy, Campus Charité Mitte, Charité - Universitätsmedizin Berlin, Berlin, Germany; ${ }^{11}$ Departments of Psychiatry and Psychology, University of Vermont, Burlington, VT, USA; ${ }^{12}$ School of Psychology, University of Nottingham, Nottingham, UK; ${ }^{13}$ Physikalisch-Technische Bundesanstalt (PTB), Braunschweig und Berlin, Germany; ${ }^{14}$ Institut National de la Santé et de la Recherche Médicale, INSERM CEA Unit 1000 "Imaging \& Psychiatry", University Paris Sud, Orsay, France; ${ }^{15}$ Rotman Research Institute, University of Toronto, Toronto, ON, Canada; ${ }^{16}$ Child Mind Institute, New York, NY, USA; ${ }^{17}$ Department of Psychiatry and Psychotherapy, Technische Universität Dresden, Dresden, Germany; ${ }^{18}$ Department of Psychology, University College Dublin, Dublin, Ireland; ${ }^{19}$ Division of Biological Research on Drug Dependence, Department of Pharmaceutical Biosciences, Uppsala University, Uppsala, Sweden
\end{abstract}

Background: Nociceptin is a key regulator linking environmental stress and alcohol drinking. In a genome-wide methylation analysis, we recently identified an association of a methylated region in the OPRL 1 gene with alcohol-use disorders. Methods: Here, we investigate the biological basis of this observation by analysing psychosocial stressors, methylation of the OPRL1 gene, brain response during reward anticipation and alcohol drinking in 660 fourteenyear-old adolescents of the IMAGEN study. We validate our findings in marchigian sardinian (msP) alcohol-preferring rats that are genetically selected for increased alcohol drinking and stress sensitivity. Results: We found that low methylation levels in intron 1 of OPRL1 are associated with higher psychosocial stress and higher frequency of binge drinking, an effect mediated by $O P R L 1$ methylation. In individuals with low methylation of $O P R L 1$, frequency of binge drinking is associated with stronger BOLD response in the ventral striatum during reward anticipation. In msP rats, we found that stress results in increased alcohol intake and decreased methylation of OPRL1 in the nucleus accumbens. Conclusions: Our findings describe an epigenetic mechanism that helps to explain how psychosocial stress influences risky alcohol consumption and reward processing, thus contributing to the elucidation of biological mechanisms underlying risk for substance abuse. Keywords: OPRL1 methylation; stressful life events; adolescence; binge drinking; nucleus accumbens.

\section{Introduction}

Adolescence is a critical period for brain development and maturation. It is characterised by changes in synaptic density and connectivity, fine-tuning of neurotransmitter systems and integration between brain structures that together lead to increased efficiency of neural function (Keshavan, Giedd, Lau, Lewis, \& Paus, 2014). However, as a consequence of these dynamic changes the adolescent brain is

\section{www.imagen-europe.com}

Conflict of interest statement: See acknowledgements for disclosures. particularly vulnerable to the exposure to traumatic life events (e.g. physical and psychological abuse) but also to more common life events (e.g. parental divorce, difficulties with authority and body changes) that are perceived as stressful by adolescents (Loth et al., 2014). Early exposure to such psychosocial stressors has been consistently associated with hazardous drinking behaviours in adolescence (Arpawong et al., 2015) that in turn predict increased risk for alcohol-use disorders in adulthood (Nees et al., 2012; Palmer et al., 2009).

Nociceptin/Orphanin FQ (N/OFQ) is a 17 amino acid peptide, that binds to its cognate $G$ proteincoupled receptor (GPCR) Opioid Receptor Like-1 
(OPRL1) also known as NOP (Meunier et al., 1995; Reinscheid et al., 1995). Several animal studies, mostly conducted on marchigian sardinian (msP) rats, an animal line genetically selected for excessive alcohol drinking and preference, have shown that activation of NOP by N/OFQ or by selective synthetic agonists reduces alcohol drinking and blunt withdrawal symptoms and according to the alcohol intoxication history and withdrawal time course, it results in anxiolytic or anxiogenic effects (Witkin et al., 2014), indicating a complex effect of N/OFQ on the regulation of anxiety and alcohol drinking.

In a recent genome-wide methylation analysis of monozygotic discordant twins, we have identified a genome-wide significant association of alcohol-use disorders with a differentially methylated region in the gene body of OPRL 1 (Ruggeri et al., 2015). Others reported an association of the promoter OPRL1 methylation with child adversity both in alcoholuse disorder cases and healthy controls (Zhang, Wang, Kranzler, Zhao, \& Gelernter, 2013). These data suggest that the N/OFQ system could be mediating alcohol problems induced by early exposure to psychosocial stress.

We hypothesise that changes in the methylation profile of OPRL1 mediate the effect of early stress exposure on hazardous drinking behaviour in adolescents by modulating the processing of rewarding stimuli. To test our hypothesis, we assessed the association of the previously identified differentially methylated region (DMR) in intron 1 (Ruggeri et al., 2015) with early stressful life events and binge drinking. We also measured its interaction with ventral striatal response during reward anticipation in a functional neuroimaging epigenetics dataset of 660 adolescents from the IMAGEN study (Schumann et al., 2010). To obtain mechanistic information and to evaluate the translational value of our results, we then assessed brain Oprl1 methylation changes induced by early stress in alcohol-preferring msP rats.

\section{Method \\ IMAGEN study participants}

We selected a randomly picked subset of 660 fourteen-year-old adolescents $(50.8 \%$ female; Table 1$)$ of the IMAGEN study, a European multicentre imaging genetic/epigenetic study. Participants were tested in eight IMAGEN assessment centres. The study was approved by local ethics research committees at each site. Written informed consent was obtained from all participants and from their legal guardians. A detailed description of recruitment and assessment procedures as well as inclusion/exclusion criteria has previously been published (Schumann et al., 2010). Behavioural information, blood and an fMRI scan were collected during a single visit to the recruitment centre when participants were 14 years old.

\section{Behavioural characterisation}

Behavioural interviews were presented by Psytools software. Lifetime stressful life events were measured with an adapted
Table 1 Demographics of the IMAGEN participants

\begin{tabular}{lcc}
\hline \multicolumn{2}{c}{ Sample size } & $\%$ \\
\hline Males & 325 & 49.2 \\
\hline & Mean & SE \\
\hline $\begin{array}{l}\text { ESPAD-Lifetime frequency of } \\
\text { binge drinking }(n=606)\end{array}$ & 0.58 & 0.05 \\
\begin{tabular}{l} 
LEQ-Lifetime stressful events $(n=626)$ \\
\hline
\end{tabular} & 6.63 & 0.11 \\
\hline
\end{tabular}

SE, standard error; ESPAD, European School Survey Project on Alcohol and Other Drugs; LEQ, life event questionnaire. ESPAD scores are on a five-level ordinal scale. Stressful life events are the sum of 20 events.

version of the Life Events Questionnaire (Newcomb, Huba, \& Bentler, 1981) and were defined based on the quality ratings of adolescents who had experienced the event (Table S1) as previously described (Loth et al., 2014). Data about lifetime frequency of binge drinking at the age of 14 were obtained from a five Likert scale question of the European School Survey Project on Alcohol and Other Drugs (ESPAD; Hibell, Centralförbundet för Alkohol-OCH Narkotikaupplysning, \& European School Survey Project on Alcohol and Other Drugs 2004). Data quality was controlled by context checks administered at the start of each task.

\section{OPRL1 methylation analysis}

DNA was extracted from whole blood and was bisulphite treated using the EZ 96 DNA methylation kit, and DNA methylation of OPRL1 was assessed using bisulphite-PCR and the Sequenom EpiTYPER system (Table S2). The signal of two $\mathrm{CpG}$ sites, located in the first intron of the OPRL1 gene and in the shelf of a CpG island (CGI; chr20:6271847362719016, hg19) previously found associated with alcohol-use disorders (Ruggeri et al., 2015), was studied. The Sequenom technology provided a mean signal for the two $\mathrm{CpG}$ sites.

\section{OPRL1 genotype analysis}

Genotype information was collected for 582,982 markers using DNA extracted from whole blood and the Illumina HumanHap610 Genotyping BeadChip. Genotype data of 11 SNPs contained in OPRL1 gene (rs1335579, rs6062342, rs6090041, rs7271530, rs2229205, rs13036542, rs4809401, rs35368587, rs910883, rs3003155, rs13039817) were extracted and tested for association with OPRL1 methylation profile. Data for rs6010718, previously associated with alcohol-use disorders (Huang, Young, Pletcher, Heilig, \& Wahlestedt, 2008), were imputed as previously described and tested for association with OPRL 1 methylation.

\section{OPRL1 gene expression analysis}

Total RNA was extracted from whole blood cells collected at the age of 14. Gene expression profiling was performed using Illumina HumanHT-12 v4 Expression BeadChips as previously described (Ruggeri et al., 2015)

\section{Monetary incentive delay task}

The participants performed a modified version of the Monetary Incentive Delay task to study neural responses to reward anticipation and reward outcome where candies were used as reward instead of money as previously described (Nees et al., 2012)

(C) 2017 Association for Child and Adolescent Mental Health. 


\section{Functional magnetic resonance imaging data acquisition and analysis}

Structural and functional magnetic resonance imaging (fMRI) data were acquired at eight IMAGEN assessment sites with 3TMRI scanners of different manufacturers. Full details of MRI acquisition protocols and quality checks have been described previously, including standardisation across MRI scanners (Schumann et al., 2010). We extracted regions of interest (ROIs) using the Marsbar toolbox (http://marsbar.sourcef orge.net). We used the left and right ventral striatum as ROIs and extracted mean blood-oxygen-level-dependent (BOLD) responses in 9-mm spheres centred at $x, y, z= \pm 15,-9,9$ in Montreal Neurological Institute space. These coordinates were based on previous findings (Yacubian et al., 2006).

\section{Animals}

Genetically selected msP male rats weighing 250-300 g (78 weeks old) at the beginning of the experiments were used (Ciccocioppo et al., 2006) and were kept in controlled conditions. All the procedures were conducted in adherence with the European Community Council Directive for Care and Use of Laboratory Animals.

\section{Early stress exposure}

A total of $32 \mathrm{msP}$ rats were subdivided in two groups, a control group that remained in the home cage throughout the procedure and a treated group receiving one of the following stressful procedure, every 7 days for 3 weeks, acute restrain stress (Ciccocioppo et al., 2014), forced swimming stress (Guan, Shao, Xie, Chen, \& Wang, 2014) and sleep deprivation stress (Youngblood, Zhou, Smagin, Ryan, \& Harris, 1997). One week after the last exposure to stress, the two groups were divided in two subgroups $(n=8)$, one stressed and one control group were killed, brains were dissected and the nucleus accumbens was frozen in dry ice and stored at $-80^{\circ} \mathrm{C}$. The other two subgroups were tested for voluntary EtOH intake for a total of 7 days and 1 week after the last exposure to alcohol.

\section{Voluntary EtOH intake}

To test voluntary EtOH intake, we used the two-bottle choice test in msP rats as previously described (Ciccocioppo et al., 2006). Briefly, animals were exposed to an intermittent $10 \%$ (v/v) ethanol solution for seven consecutive days. Alcohol was offered $24 \mathrm{~h}$ a day. Alcohol, water and food consumption were monitored $24 \mathrm{hr}$ from the moment that EtOH was made available. Food intake was measured by weighing the food containers and taking into account spillage.

\section{Oprl1 methylation and gene expression analysis}

DNA and RNA were simultaneously extracted from the left and right nucleus accumbens. DNA was bisulphite treated using the EZ DNA methylation kit, and DNA methylation of Oprl1 was assessed using bisulphite-PCR and the Sequenom EpiTYPER system (Table S2). Gene expression of Oprl1 and the housekeeping gadph were measured via quantitative polymerase chain reaction (Table S2). The ABI Prism ${ }^{\circledR}$ SDS 2.1 software was used to analyse the specificity (dissociation curve) and relative quantification of the Oprl1 amplicon $(\Delta \mathrm{Ct})$.

\section{Statistical analyses}

The general linear model was used to determine associations among human OPRL1 methylation and OPRL1 genotype and gene expression, negative life events, drinking behaviour and BOLD responses correcting for gender and recruitment site (handiness was corrected for BOLD responses).

A mediation analysis was carried out to test if the OPRL1 methylation mediated the association of lifetime stressful events and frequency of binge drinking using PROCESS (Hayes, 2013), a SPSS macro that uses a path analytical framework for estimating direct and indirect effects based on OLS regression models. This approach involves bootstrapping the sampling distribution of the indirect effect and obtaining its confidence interval. The analysis is based on 5,000 bootstraps with stressful events as a predictor variable, OPRL1 methylation as a mediator, and binge drinking as the dependent variable.

Methyl-eQTL analysis was performed using the $R$ package ( $R$ version 3.1.2) Matrix expression QTL (Shabalin, 2012). The analysis was performed on 34,833 gene expression probes and OPRL 1 methylation data generated on the Sequenom platform. We run a linear regression model adjusted for gender and recruitment centre.

The database for annotation, visualisation and integrated discovery (DAVID; Huang, Sherman, \& Lempicki, 2009) was used to determine enrichment of biological processes and disease classes in the gene list generated from the methyl-eQTL analysis with $p<.05$. Enrichment for specific biological functions and disease classes was determined using a modified Fisher's test at a significance level of $p<.05$.

The effect of the interaction of OPRL1 methylation and ventral striatum BOLD response on frequency of binge drinking was tested with a general linear model.

A principal component analysis was applied on the methylation data collected across the rat Oprl1 gene, where the missing data were replaced by means and a varimax rotation was also applied to increase interpretability of outcomes. Components with eigenvalue $>1$ were selected for further analyses.

All analyses were carried out using the software IBM SPSS statistics (version 22) unless otherwise stated. $p$-values corrected for multiple testing are reported as $p_{\text {corrected. }}$

\section{Results}

\section{OPRL1 methylation mediates the association of early exposure to psychosocial stress and frequency of binge drinking at the age of 14}

We found a negative association of OPRL1 methylation and lifetime frequency of binge drinking in participants aged 14 years $(t=-3.56, p=2.2 \times$ $10^{-04}, \eta_{\mathrm{p}}^{2}=.021$; Figure $1 \mathrm{~A}$, Table 1). We then investigated whether lifetime stressful events were associated with lifetime frequency of binge drinking and with OPRL1 methylation. We found a positive association of stressful life events and lifetime binge drinking at the age of $14\left(t=4.56, p=8.7 \times 10^{-06}\right.$, $\eta_{\mathrm{p}}^{2}=.036$; Figure $\left.1 \mathrm{~B}\right)$ and a negative association of stressful life events with OPRL1 methylation $\left(t=-2.50, \quad p=.013, \quad \eta_{\mathrm{p}}^{2}=.010 ; \quad\right.$ Figure 1C). A subsequent mediation analysis revealed that the indirect effect of psychosocial stressors on binge drinking through OPRL1 methylation was significant $(z=2.17, p=0.030)$ and that the 95\% confidence interval did not include 0 (95\% CI [0.002, $0.019])$.

To investigate if OPRL1 methylation is dependent on OPRL1 genotype, we used a general linear model 

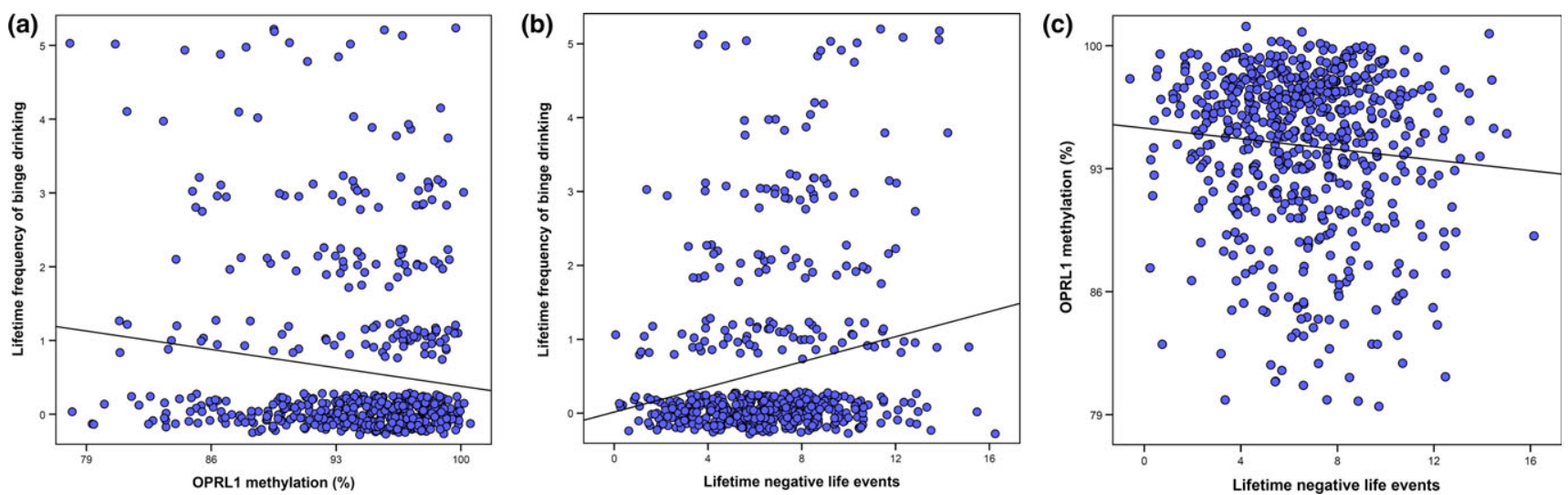

Figure 1 Association of OPRL1 methylation with lifetime frequency of binge drinking $(N=609)$ and stressful life events $(N=626)$ in adolescents. A, Negative association of OPRL1 methylation and lifetime frequency of binge drinking $\left(p=2.2 \times 10^{-04}\right)$. B, Positive association of stressful life events and lifetime frequency of binge drinking $\left(p=8.7 \times 10^{-06}\right)$ in 14 -year-old adolescents. $C$, Negative association of lifetime frequency of binge drinking and OPRL1 methylation $(p=.013)$

to test 12 SNPs contained in the OPRL1 gene, including rs6010718, which was previously associated with alcohol-use disorders (Huang et al., 2008). We found no significant association between the OPRL 1 methylation profile and any of the SNPs taken in consideration; neither we found any significant association of SNPs and lifetime binge drinking (Table S3).

\section{OPRL1 methylation and gene expression}

To explore the relationship between OPRL1 methylation and gene expression, we performed a methyleQTL analysis and we found a total of 1,856 probes mapping 1,245 genes associated with OPRL 1 methylation $(p<.05$; Table S4); no OPRL 1 cis-gene expression was observed. We investigated whether common pathways, biological functions or pathologies were overrepresented in the list of 1,245 genes associated to OPRL1 methylation with $p<.05$. The analysis using DAVID, a database and tool for functional annotation (Huang et al., 2009), revealed enrichment for phenotypically relevant gene networks, including the 'disorder categories', 'neurological' and 'psychological' disorders ( $p=.003, n=48$; $p=.004, n=46$, respectively) and genes involved in dopamine, acetylcholine and glutamate signalling (Tables S5 and S6).

\section{Ventral striatal activation during reward anticipation is associated with frequency of binge drinking and interacts with OPRL1 methylation}

The reinforcing properties of alcohol are dependent on activation of the ventral striatum, a brain area, which is central to reward processing, including reward anticipation. We measured reward anticipation in the Monetary Incentive Delay task in 393 14year-old IMAGEN participants where neuroimaging, methylation and drinking data were available, and found a positive association of frequency of binge drinking with BOLD response in the left ventral striatum $\left(t=2.87, \quad p=.004, \quad p_{\text {corrected }}=.008\right.$, $\left.\eta_{\mathrm{p}}^{2}=.022\right)$ and the right ventral striatum $(t=2.33$, $\left.p=.020, p_{\text {corrected }}=.040, \eta_{\mathrm{p}}^{2}=.015\right)$. We found no significant association of OPRL1 methylation and BOLD response in the left ventral striatum $(t=2.02$, $\left.p=.044, p_{\text {corrected }}=.088, \eta_{\mathrm{p}}^{2}=.010\right)$ and the right ventral striatum $\left(t=1.86, p=.064, p_{\text {corrected }}=.128\right.$, $\left.\eta_{\mathrm{p}}^{2}=.009\right)$ after correction for multiple comparison. Since both OPRL1 methylation and ventral striatal response during reward anticipation are associated with binge drinking, we investigated if there was an interaction of these variables on binge drinking. We found a significant interaction between OPRL1 methylation and the left ventral striatum activation $\left(t=-2.69, p=.007, p_{\text {corrected }}=.014, \eta_{\mathrm{p}}^{2}=.020 ;\right.$ Figure $2 \mathrm{~B}$ and $2 \mathrm{D})$ and a trend in the right ventral striatum $\left(t=-2.18, \quad p=.030, \quad p_{\text {corrected }}=.060\right.$, $\eta_{\mathrm{p}}^{2}=.013$; Figure $2 \mathrm{~A}$ and $2 \mathrm{C}$ ). Our data suggest that the association of ventral striatum activation and frequency of binge drinking is observed only in individuals with low OPRL1 methylation levels.

\section{Early stress and differential methylation are associated with oprl1 gene expression in the nucleus accumbens of $\mathrm{ms} P$ rats}

We next investigated if the relation between psychosocial stress, alcohol abuse and OPRL1 methylation in peripheral blood can be validated in the ventral striatum, a brain structures that is central to reward processing and anticipation. It is not possible to obtain postmortem brain materials of the ventral striatum from a sufficient number of human adolescents to carry out association analyses; we, therefore, used an established animal model, namely msP rats, for our experiments. We exposed msP rats to a stress protocol and, similar to humans, found a significant overall effect of stress exposure on voluntary alcohol intake $(F(1,13)=45.05, p<.0001$; Figure 3$)$. Water and food consumption were not significantly affected 

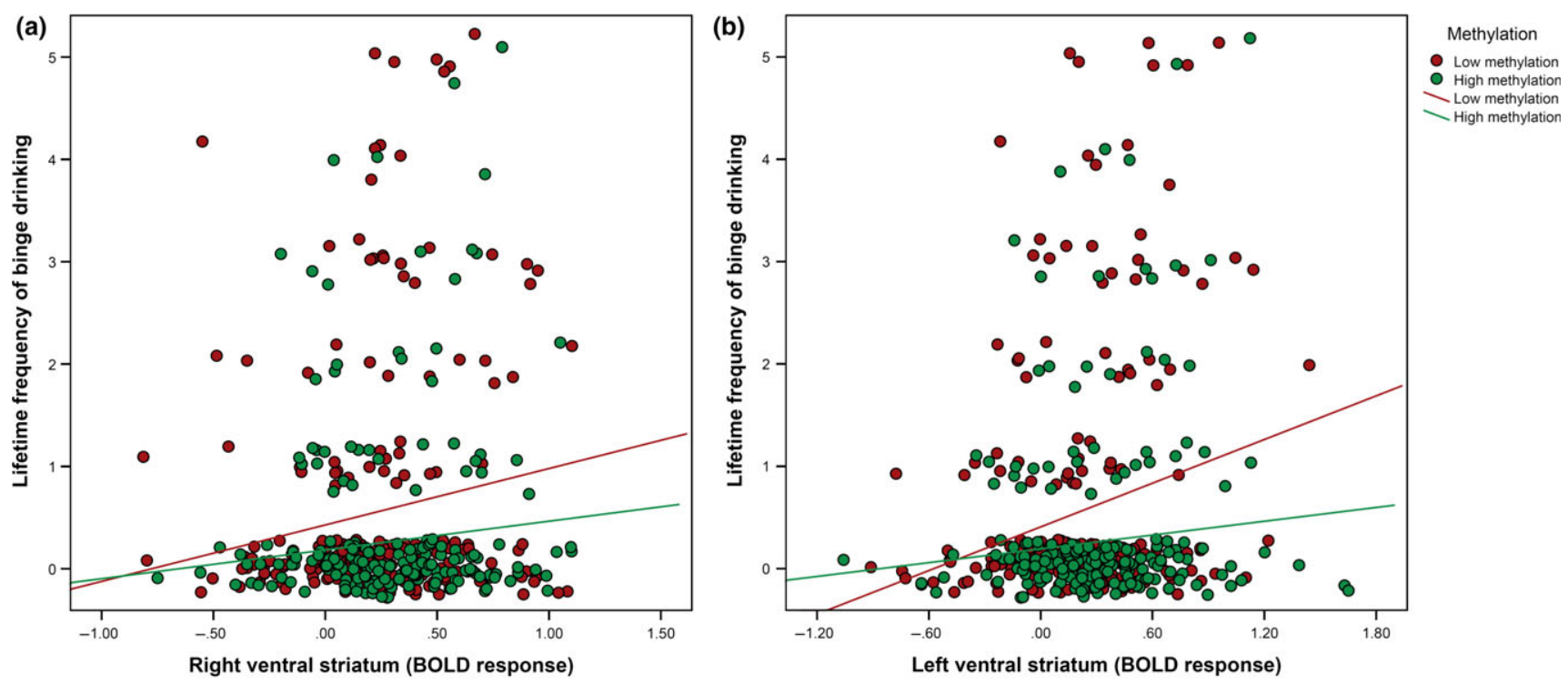

(c)
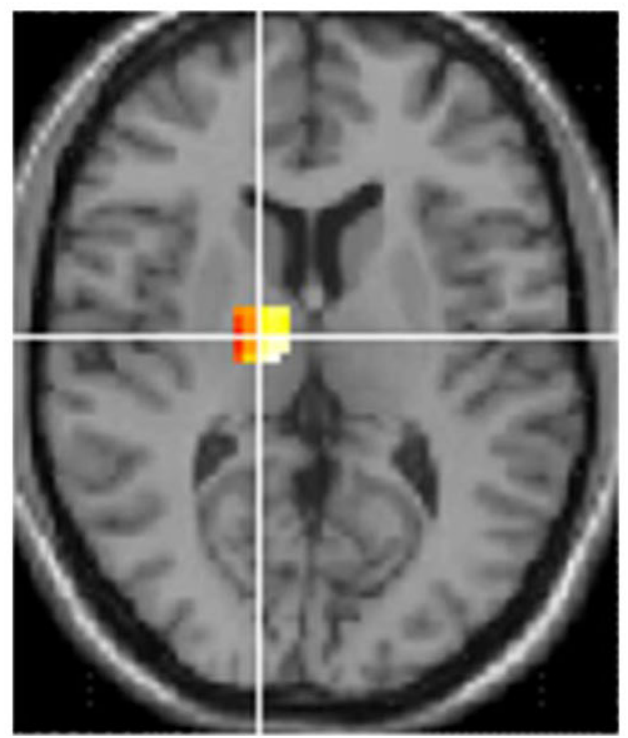

(d)

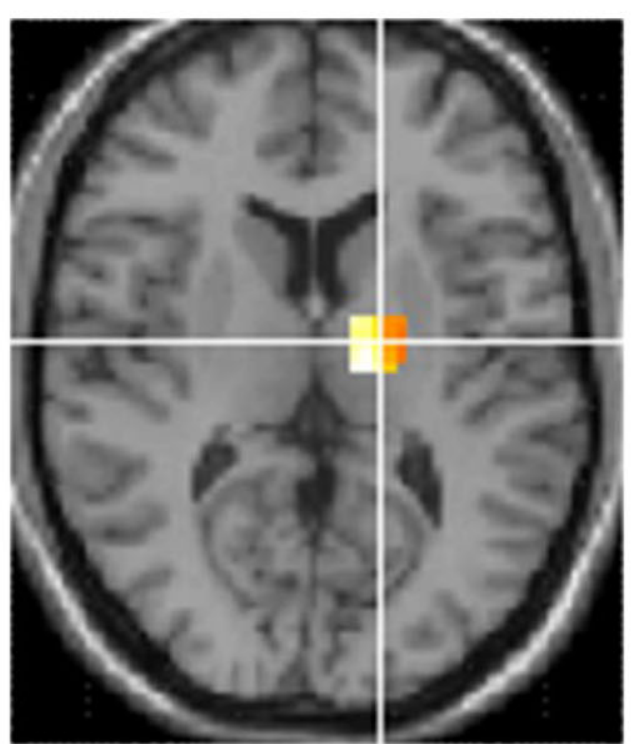

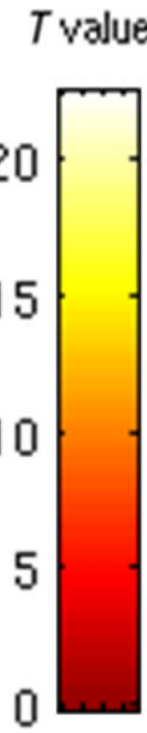

Figure 2 Association of activation of the ventral striatal activation during Monetary Incentive Delay Task and lifetime binge drinking in adolescents $(N=393)$ and interaction with OPRL1 methylation. Significant interaction between OPRL1 methylation and the left VS activation $\left(p=.007, p_{\text {corrected }}=.014\right)$ while the interaction was only a trend in the right VS $\left(p=.030, p_{\text {corrected }}=.060\right)$. Methylation values in panels A and B are separated into low and high methylation using a median slip. Coronal section shows methylation differences in right and left ventral activation during reward anticipation $(x, y, z: \pm 9,11,-2$; panels $C$ and $D)$

by stress exposure (Tables $\mathrm{S} 7$ and $\mathrm{S} 8$ ). In stressed versus nonstressed animals, we then compared Oprl1 methylation and gene expression levels in the nucleus accumbens, the core region of the ventral striatum (Galtress \& Kirkpatrick, 2010). We assessed methylation levels of the entire Oprl1 gene locus and detected 39 methylation sites. We then carried out a principal component analysis of these sites identifying 11 principal components (eigenvalue $>1$ ) that explained $82 \%$ of the variance (Table S9). An exploratory analysis in stressed animals revealed a significant association of Oprl1 gene expression and one methylation component explaining $8 \%$ of the variance $(r=.799, \quad p=.001, n=13) ;$ no association was observed in nonstressed animals $(r=.054, p=.861$, $n=13$ ). A subsequent moderation analysis showed an interaction effect of stress and methylation on gene expression of Oprl1 in the nucleus accumbens $(z=2.33, p=.020$; two-tailed test; Figure S1).

\section{Discussion}

In a population-based sample of 14-year-old adolescents of the IMAGEN cohort (Schumann et al., 2010), we showed that decreased methylation in the first intron of OPRL1 gene is associated with increased early psychosocial stress exposure and higher frequency of binge drinking, a risk factor for future alcohol-use disorders (Nees et al., 2012). Among individuals with lower methylation of OPRL1, we found association of high frequency of binge drinking with increased BOLD response in the 


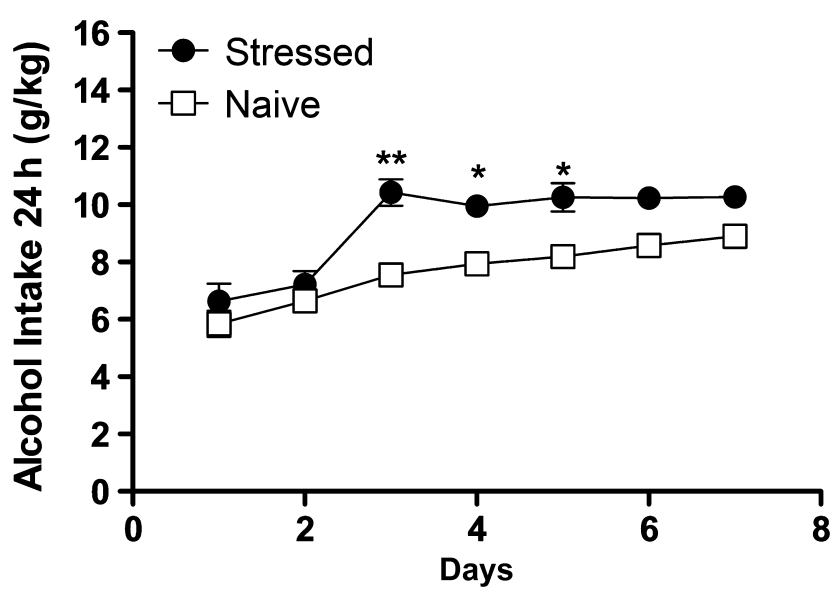

Figure 3 Association of early stress and intermittent alcohol intake in the msP rats. One-way ANOVA revealed a significant overall effect of the stress exposure on voluntary alcohol intake in msP rats $(p<.0001)$. The effect appeared from the third day of alcohol exposure and remained significant on the fourth and fifth day of alcohol exposure as shown by Newman-Keuls tests. $* p<.05$

ventral striatum during reward anticipation, reflecting enhanced reward sensitivity. The OPRL1 gene encodes for the N/OFQ receptor (NOP), a G proteincoupled receptor that together with its ligand N/OFQ has been widely studied for its role in alcohol-related behaviour both in humans and in animal models (Witkin et al., 2014). Providing corroborative evidence in $\mathrm{msP}$ rats, an animal model of innate vulnerability to excessive drinking and high sensitivity to stress (Witkin et al., 2014), we show that stress is associated with increased alcohol intake of these animals and interacts with a component of differentially methylated regions resulting in association with altered gene expression of the Oprl1 in the nucleus accumbens.

The ventral striatum and nucleus accumbens are subcortical areas involved in reward processing that are heavily innervated by mesolimbic dopamine neurons (Tritsch \& Sabatini, 2012). Increased BOLD response in this region may reflect an increase dopaminergic activity leading to enhanced sensitivity to reward. However, striatal activity is also influenced by other neurocircuitries, including glutamatergic efferents from the basolateral amygdala and the frontal cortex (Fudge, Kunishio, Walsh, Richard, \& Haber, 2002; Haber, Kim, Mailly, \& Calzavara, 2006). The NOP receptor is functionally coupled to inhibition of adenylate cyclase, activation of MAP kinase, activation of $\mathrm{K}^{+}$conductance, and inhibition of $\mathrm{Ca}^{+2}$ conductance and induces inhibition of neurotransmitter release, including of dopamine, glutamate, gammaamino butyric acid (GABA), acetylcholine and others (Ciccocioppo, Panocka, Polidori, Regoli, \& Massi, 1999). NOP receptor activity, thus, results in a comprehensive direct and indirect regulatory system of reward processing (Neal et al., 1999). Our observation of a transregulation of genes involved in dopamine and glutamate signalling by OPRL1 methylation in human blood is in keeping with these findings.
Previous studies, including our own findings in adult alcohol abusing discordant twins, reported a positive association between OPRL1 hypermethylation and alcohol-use disorders (Ruggeri et al., 2015), as well as a positive association with child adversity in both alcohol-use disorders cases and healthy controls (Zhang et al., 2013). In addition to the severity of the environmental stressor, we looked at stressful events, while in a previous study they investigated severe physical and emotional abuse (Ruggeri et al., 2015; Zhang et al., 2013), the most striking difference of our study is that we investigated adolescents at age 14 years where binge drinking is less intense and frequent as in adult alcohol abusers. In fact, it is a reflection of personality traits involving anxiety sensitivity, sensation seeking and/or impulsiveness (Nees et al., 2012; Woicik, Stewart, Pihl, \& Conrod, 2009). While these traits are associated with psychosocial stress and alcohol drinking (Whelan et al., 2014), our young participants have not yet developed alcohol-use disorders neither have they been exposed to protracted consumption of alcohol that is known to alter the methylation profile (Weng, Wu, Lee, Hsu, \& Cheng, 2015). Possibly OPRL1 hypomethylation may occur as a result of adverse life events and may contribute to enhanced reward sensitivity and propensity to binge drinking. This epigenetic change may subsequently be reversed following protracted exposure to alcohol, and possibly contribute to decreased striatal activation often observed in patients suffering from alcohol-use disorders (Chen et al., 2011).

Epigenetic regulation of gene expression can be species-specific (Zeng et al., 2012) and is contingent on several factors, including epigenetic imprinting (Adalsteinsson \& Ferguson-Smith, 2014), biological age (Talens et al., 2012) and tissue specificity (Grunau, Hindermann, \& Rosenthal, 2000). It is a limitation of our study that in humans, we were only able to measure methylation and gene expression in peripheral blood, as there are no sufficient adolescent postmortem brain data from human ventral striatum available to analyse this brain region. A validation in the brain of the stress-induced OPRL1 hypomethylation found in the human blood was provided by our findings in the nucleus accumbens of stressed msP rats, which showed not only a similar association of stress and Oprl1 methylation but also an association between Oprl1 methylation and its gene expression.

Methylation in the promoter area is often negatively correlated with gene expression; however, correlation of methylation in the gene body and cisgene expression does not show a specific directionality (Lee, Choi, Lee, \& Kim, 2015). Our findings show that stress-induced hypomethylation of the Oprl1 gene body in $\mathrm{msP}$ rats is associated with a decrease in Oprl1 gene expression. It is possible that a similar correlation between OPRL1 methylation 
and gene expression exists in human brain, consistent with the decreased level of OPRL1 mRNA found in the brain of alcohol-use disorders patients (Kuzmin et al., 2009).

A previous study reported an association of the OPRL 1 genetic variant rs6010718 in intron 1 with alcohol-use disorders (Huang et al., 2008). There is evidence that the methylation of certain $\mathrm{CpG}$ sites is correlated with single-nucleotide polymorphisms (SNPs; Shoemaker, Deng, Wang, \& Zhang, 2010), suggesting that an interplay of genetic and epigenetics mechanisms could underlie complex disorder. The absence of association in our data suggests independence of genetic and epigenetic mechanisms related to alcohol drinking in this gene locus.

Together our findings describe an epigenetic mechanism that helps to explain how psychosocial stress influences risky alcohol consumption and reward processing, thus contributing to the elucidation of biological mechanisms underlying risk for substance abuse.

\section{Supporting information}

Additional Supporting Information may be found in the online version of this article:

Table S1. Frequency of stressful life events.

Table S2. Human and rat OPRL1 methylation and rat oprl1 gene expression assessment.

Table S3. Association of OPRL1 genetic variants and OPRL1 methylation or lifetime binge drinking.

Table S4. List of OPRL 1 methyl-eQTL in the blood of 14year-old adolescents.

Table S5. Enriched disease categories $(p<.05)$ for OPRL1 methyl-eQTLs in blood of 14-year-old adolescents.

Table S6. Enriched GO biological processes categories $(p<.05)$ for OPRL1 methyl-eQTLs in blood of 14-year-old adolescents.

Table S7. PCA loadings of $39 \mathrm{CpG}$ sites of the rat oprl1 gene $(N=16)$.

Table s8. Water consumption (in $\mathrm{g} / \mathrm{kg}$ ) of stressed versus naive rats during intermittent $10 \%$ alcohol exposure.

Table s9. Food consumption (in $\mathrm{g} / \mathrm{kg}$ ) of stressed versus naive rats during intermittent $10 \%$ alcohol exposure.

Figure S1. The scatter plot of rat Oprl1 methylation factor and Oprl1 gene expression conditioned on the status of stress in the nucleus accumbens.

\section{Acknowledgements}

This work received support from the following sources: the European Union-Funded FP6 Integrated Project IMAGEN (LSHM-CT- 2007-037286), the FP7 projects IMAGEMEND (602450), AGGRESSOTYPE (602805) and MATRICS (603016), the Innovative Medicine Initiative Project EU-AIMS (115300-2), the Medical Research Council Grant 'Developmental Pathways into Adolescent Substance Abuse' (93558) and Consortium on Vulnerability to Externalizing Disorders and Addictions [c-VEDA] (MR/N000390/1), the Swedish Funding Agencies VR, FORTE and FORMAS, the Medical Research Council and the Wellcome Trust (Behavioural and Clinical Neuroscience Institute, University of Cambridge), the National Institute for Health Research (NIHR) Biomedical Research Centre at South London and Maudsley NHS Foundation Trust and King's College London, the Bundesministeriumfür Bildung und Forschung (BMBF grants 01GS08152; 01EV0711; eMED SysAlc01ZX1311A; Forschungsnetz AERIAL), the Deutsche Forschungsgemeinschaft (DFG grants SM 80/7-1, SM 80/7-2, SFB 940/1), the National Institutes of Health, USA (Axon, Testosterone and Mental Health during Adolescence; RO1 MH08577201A1), and by NIH Consortium grant U54 EB020403, supported by a cross-NIH alliance that funds Big Data to Knowledge Centres of Excellence. TB has served as an advisor or consultant to Bristol-Myers Squibb, Desitin Arzneimittel, Eli Lilly, Medice, Novartis, Pfizer, Shire, UCB and Vifor Pharma; he has received conference attendance support, conference support or speaking fees from Eli Lilly, Janssen McNeil, Medice, Novartis, Shire and UCB; and he is involved in clinical trials conducted by Eli Lilly, Novartis and Shire; the present work is unrelated to these relationships. JG has received research funding from the German Federal Ministry of Education and Research, AstraZeneca, Eli Lilly, Janssen-Cilag and Bristol-Myers Squibb; he has received speaking fees from AstraZeneca, JanssenCilag and Bristol-Myers Squibb. The remaining authors have declared that they have no competing or potential conflicts of interest.

\section{Correspondence}

Gunter Schumann, MRC-SGDP Centre, Institute of Psychiatry, Psychology and Neuroscience, King's College London, De Crespigny Park, London SE5 8AF, UK; Email: gunter.schumann@kcl.ac.uk

\section{Key points}

- Nociceptin is a key regulator linking environmental stress and alcohol drinking and methylation of the OPRL1 gene is associated with alcohol-use disorders.

- We investigate the biological basis of this observation by analysing psychosocial stressors, methylation of the OPRL1 gene and brain response during reward anticipation and alcohol drinking in 660 fourteen year-old adolescents of the IMAGEN study.

- We found that low levels of OPRL1 methylation mediates the association of psychosocial stress and frequency of binge drinking; in individuals with low methylation of OPRL 1 frequency of binge drinking is associated with stronger BOLD-response in the ventral striatum during reward anticipation. 
- In Marchigian-Sardinian alcohol-preferring rats that are genetically selected for increased alcohol drinking and stress sensitivity we validated our findings by showing that stress results in increased alcohol intake and decreased methylation of OPRL1 in the nucleus accumbens.

- We describe for the first time how psychosocial stressors relate to epigenetic methylation of a well-established candidate gene, and how this relation influences brain activity and risky drinking behavior in adolescents; importantly, we provide parallel evidence of a similar epigenetic regulation in brain tissue of a relevant animal model.

\section{References}

Adalsteinsson, B.T., \& Ferguson-Smith, A.C. (2014). Epigenetic control of the genome-lessons from genomic imprinting. Genes (Basel), 5, 635-655.

Arpawong, T.E., Sussman, S., Milam, J.E., Unger, J.B., Land, H., Sun, P., \& Rohrbach, L.A. (2015). Post-traumatic growth, stressful life events, and relationships with substance use behaviors among alternative high school students: A prospective study. Psychology and Health, 30, 475-494.

Chen, G., Cuzon Carlson, V.C., Wang, J., Beck, A., Heinz, A., Ron, D., .. \& \& Buck, K.J. (2011). Striatal involvement in human alcoholism and alcohol consumption, and withdrawal in animal models. Alcoholism: Clinical and Experimental Research, 35, 1739-1748.

Ciccocioppo, R., Economidou, D., Cippitelli, A., Cucculelli, M., Ubaldi, M., Soverchia, L., .. \& \& Massi, M. (2006). Genetically selected Marchigian Sardinian alcohol-preferring (msP) rats: An animal model to study the neurobiology of alcoholism. Addiction Biology, 11, 339-355.

Ciccocioppo, R., Panocka, I., Polidori, C., Regoli, D., \& Massi, M. (1999). Effect of nociceptin on alcohol intake in alcoholpreferring rats. Psychopharmacology (Berl), 141, 220-224.

Ciccocioppo, R., Stopponi, S., Economidou, D., Kuriyama, M., Kinoshita, H., Heilig, M., .. \& \& Teshima, K. (2014). Chronic treatment with novel brain-penetrating selective NOP receptor agonist MT-7716 reduces alcohol drinking and seeking in the rat. Neuropsychopharmacology, 39, 2601-2610.

Fudge, J.L., Kunishio, K., Walsh, P., Richard, C., \& Haber, S.N. (2002). Amygdaloid projections to ventromedial striatal subterritories in the primate. Neuroscience, 110, 257-275.

Galtress, T., \& Kirkpatrick, K. (2010). The role of the nucleus accumbens core in impulsive choice, timing, and reward processing. Behavioral Neuroscience, 124, 26-43.

Grunau, C., Hindermann, W., \& Rosenthal, A. (2000). Largescale methylation analysis of human genomic DNA reveals tissue-specific differences between the methylation profiles of genes and pseudogenes. Human Molecular Genetics, 9, 2651-2663.

Guan, X.T., Shao, F., Xie, X., Chen, L., \& Wang, W. (2014). Effects of aspirin on immobile behavior and endocrine and immune changes in the forced swimming test: Comparison to fluoxetine and imipramine. Pharmacology Biochemistry and Behavior, 124, 361-366.

Haber, S.N., Kim, K.S., Mailly, P., \& Calzavara, R. (2006). Reward-related cortical inputs define a large striatal region in primates that interface with associative cortical connections, providing a substrate for incentive-based learning. Journal of Neuroscience, 26, 8368-8376.

Hayes, A.F. (2013). Introduction to mediation, moderation, and conditional process analysis a regression-based approach. New York: Guilford Press.

Hibell, B., Centralförbundet för Alkohol- OCH Narkotikaupplysning, \& European School Survey Project on Alcohol and Other Drugs (2004). The ESPAD report 2003: alcohol and other drug use among students in 35 European countries, Stockholm: Swedish Council for Information on Alcohol and Other Drugs (Centralförb. för alkohol- och narkotikaupplysning)(CAN).
Huang, D.W., Sherman, B.T., \& Lempicki, R.A. (2009). Systematic and integrative analysis of large gene lists using DAVID bioinformatics resources. Nature Protocols, 4, 44-57.

Huang, J., Young, B., Pletcher, M.T., Heilig, M., \& Wahlestedt, C. (2008). Association between the nociceptin receptor gene (OPRL1) single nucleotide polymorphisms and alcohol dependence. Addiction Biology, 13, 88-94.

Keshavan, M.S., Giedd, J., Lau, J.Y., Lewis, D.A., \& Paus, T. (2014). Changes in the adolescent brain and the pathophysiology of psychotic disorders. Lancet Psychiatry, 1, 549-558.

Kuzmin, A., Bazov, I., Sheedy, D., Garrick, T., Harper, C., \& Bakalkin, G. (2009). Expression of pronociceptin and its receptor is downregulated in the brain of human alcoholics. Brain Research, 1305(Suppl), S80-S85.

Lee, S.M., Choi, W.Y., Lee, J., \& Kim, Y.J. (2015). The regulatory mechanisms of intragenic DNA methylation. Epigenomics, 7, 527-531.

Loth, E., Poline, J.B., Thyreau, B., Jia, T., Tao, C., Lourdusamy, A., ... \& IMAGEN Consortium (2014). Oxytocin receptor genotype modulates ventral striatal activity to social cues and response to stressful life events. Biological Psychiatry, 76, 367-376.

Meunier, J.C., Mollereau, C., Toll, L., Suaudeau, C., Moisand, C., Alvinerie, P., ... \& Mazarguil, H. (1995). Isolation and structure of the endogenous agonist of opioid receptor-like ORL1 receptor. Nature, 377, 532-535.

Neal, C.R., Mansour Jr, A., Reinscheid, R., Nothacker, H.P., Civelli, O., Akil, H., \& Watson Jr, S.J. (1999). Opioid receptor-like (ORL1) receptor distribution in the rat central nervous system: Comparison of ORL1 receptor mRNA expression with (125)I-[(14)Tyr]-orphanin FQ binding. Journal of Comparative Neurology, 412, 563-605.

Nees, F., Tzschoppe, J., Patrick, C.J., Vollstadt-Klein, S., Steiner, S., Poustka, L., .. \& \& IMAGEN Consortium (2012). Determinants of early alcohol use in healthy adolescents: The differential contribution of neuroimaging and psychological factors. Neuropsychopharmacology, 37, 986-995.

Newcomb, M.D., Huba, G.J., \& Bentler, P.M. (1981). A multidimensional assessment of stressful life events among adolescents - derivation and correlates. Journal of Health and Social Behavior, 22, 400-415.

Palmer, R.H., Young, S.E., Hopfer, C.J., Corley, R.P., Stallings, M.C., Crowley, T.J., \& Hewitt, J.K. (2009). Developmental epidemiology of drug use and abuse in adolescence and young adulthood: Evidence of generalized risk. Drug and Alcohol Dependence, 102, 78-87.

Reinscheid, R.K., Nothacker, H.P., Bourson, A., Ardati, A., Henningsen, R.A., Bunzow, J.R., . . \& Civelli Jr, O. (1995). Orphanin FQ: A neuropeptide that activates an opioid like G protein-coupled receptor. Science, 270, 792-794.

Ruggeri, B., Nymberg, C., Vuoksimaa, E., Lourdusamy, A., Wong, C.P., Carvalho, F.M., ... \& IMAGEN Consortium (2015). Association of protein phosphatase PPM1G with alcohol use disorder and brain activity during behavioral control in a genome-wide methylation analysis. American Journal of Psychiatry, 172, 543-552.

Schumann, G., Loth, E., Banaschewski, T., Barbot, A., Barker, G., Buchel, C., ... \& IMAGEN Consortium (2010). The 
IMAGEN study: Reinforcement-related behaviour in normal brain function and psychopathology. Molecular Psychiatry, $15,1128-1139$

Shabalin, A.A. (2012). Matrix eQTL: Ultra fast eQTL analysis via large matrix operations. Bioinformatics, 28, 13531358.

Shoemaker, R., Deng, J., Wang, W., \& Zhang, K. (2010). Allele-specific methylation is prevalent and is contributed by CpG-SNPs in the human genome. Genome Research, 20, 883-889.

Talens, R.P., Christensen, K., Putter, H., Willemsen, G., Christiansen, L., Kremer, D., ... \& Heijmans, B.T. (2012). Epigenetic variation during the adult lifespan: Cross-sectional and longitudinal data on monozygotic twin pairs. Aging Cell, 11, 694-703.

Tritsch, N.X., \& Sabatini, B.L. (2012). Dopaminergic modulation of synaptic transmission in cortex and striatum. Neuron, 76, 33-50.

Weng, J.T., Wu, L.S., Lee, C.S., Hsu, P.W., \& Cheng, A.T. (2015). Integrative epigenetic profiling analysis identifies DNA methylation changes associated with chronic alcohol consumption. Computers in Biology and Medicine, 64, 299306.

Whelan, R., Watts, R., Orr, C.A., Althoff, R.R., Artiges, E., Banaschewski, T., ... \& IMAGEN Consortium (2014). Neuropsychosocial profiles of current and future adolescent alcohol misusers. Nature, 512, 185-189.

Witkin, J.M., Statnick, M.A., Rorick-Kehn, L.M., Pintar, J.E., Ansonoff, M., Chen, Y., ... \& Ciccocioppo, R. (2014). The biology of Nociceptin/Orphanin FQ (N/OFQ) related to obesity, stress, anxiety, mood, and drug dependence. Pharmacology and Therapeutics, 141, 283-299.

Woicik, P.A., Stewart, S.H., Pihl, R.O., \& Conrod, P.J. (2009). The Substance Use Risk Profile Scale: A scale measuring traits linked to reinforcement-specific substance use profiles. Addictive Behaviors, 34, 1042-1055.

Yacubian, J., Glascher, J., Schroeder, K., Sommer, T., Braus, D.F., \& Buchel, C. (2006). Dissociable systems for gain- and loss-related value predictions and errors of prediction in the human brain. Journal of Neuroscience, 26, 9530-9537.

Youngblood, B.D., Zhou, J., Smagin, G.N., Ryan, D.H., \& Harris, R.B. (1997). Sleep deprivation by the "flower pot" technique and spatial reference memory. Physiology and Behavior, 61, 249-256.

Zeng, J., Konopka, G., Hunt, B.G., Preuss, T.M., Geschwind, D., \& Yi, S.V. (2012). Divergent whole-genome methylation maps of human and chimpanzee brains reveal epigenetic basis of human regulatory evolution. The American Journal of Human Genetics, 91, 455-465.

Zhang, H., Wang, F., Kranzler, H.R., Zhao, H., \& Gelernter, J. (2013). Profiling of childhood adversity-associated DNA methylation changes in alcoholic patients and healthy controls. PLoS ONE, 8, e65648.

Accepted for publication: 16 October 2017 